\title{
RECONOCIMIENTO \\ Y PERCEPCIÓN DEL REGISTRO \\ COLOQUIAL ESPAÑOL EN \\ ALUMNOS POLACOS: EL CASO \\ DE LOS PRO-VERBOS
}

\author{
Raúl Fernández Jódar \\ Universidad Adam Mickiewicz de Poznań \\ http://dx.doi.org/10.18778/8220-201-4.07
}

\section{Resumen}

En el presente texto presentamos los conceptos de reconocimiento y percepción como herramientas para la descripción de los registros formal e informal. Dichos conceptos pueden resultar de utilidad para estudiar la relación entre el español como lengua extranjera y una lengua segunda. Con ellos se pretende facilitar tanto la descripción interlingüística de los registros de un aprendiz de E/LE como el análisis contrastivo de los registros formal y coloquial. Como ejemplo, mostramos los resultados respecto al uso de los pro-verbos por parte de aprendices polacos de E/LE.

Palabras clave: Registro, español coloquial, informal, formal, pro-verbo.

1.

\section{Introducción}

Ya hace años que los prejuicios que pudiera haber entre los lingüistas respecto al registro coloquial como materia de investigación han desaparecido ${ }^{1}$. Incluso se podría creer que en las clases

1 En el ámbito de la lengua española debemos destacar la obra de A. Briz Gómez, fundador e impulsor del Grupo Val.Es.Co., el cual 
de español como lengua extranjera el registro coloquial ocupa un pequeño lugar dentro de los manuales.

Sin embargo, consideramos que no es suficiente con la descripción de ciertos aspectos pragmáticos o la mención a un limitado grupo léxico. El hecho de que no se hayan manejado herramientas que puedan describir sus usos y valores de forma cuantitativa para así contrastarlos con los de otras lenguas o los usos que llevan a cabo los hablantes de una lengua segunda, puede llevar a percepciones erróneas por parte de los aprendices.

En los siguientes apartados ejemplificamos cómo la enseñanza de una lengua extranjera puede influir en la percepción que el aprendiz tiene de ciertas características del registro coloquial.

\section{2. \\ El reconocimiento y la percepción del registro como herramientas de análisis}

En el presente estudio trabajamos con dos conceptos como son el reconocimiento y la percepción, los cuales parten, evidentemente, de la existencia del conocimiento previo del léxico analizado por parte de los sujetos estudiados. El primero de los dos conceptos se refiere a la capacidad del hablante de una lengua para enmarcar cada característica de dicha lengua en el ámbito de lo coloquial o de lo formal. El segundo, en cambio, se refiere a la capacidad del hablante para discernir el uso correcto de una determinada característica de los registros, otorgándole mayor o menor carga de uno u otro registro.

no solo ha aportado una gran cantidad de publicaciones de referencia, sino también ha llevado a cabo la creación de un corpus del español coloquial de libre consulta. Es de destacar también la obra de Gaviño Rodríguez (2008). En el ámbito polaco destacamos los trabajos de Anusiewicz (1992), Bartmiński (1991, 1992 y 1993), Hołówka (1986) y, sobre todo, de Zdunkiewicz-Jedynak (2008). 
Tengamos ahora en cuenta las palabras galleta, torta y mamporro, todas ellas recogidas en el DRAE como sinónimas de bofetada y descritas como coloquiales. Pero, ¿poseen el mismo grado de coloquialidad? Un usuario de la lengua tiene la capacidad de reconocerlas como coloquiales. Sin embargo, no por ello les otorgaría el mismo nivel de coloquialidad, por lo que no las utilizaría en los mismos contextos. Es decir, el usuario de la lengua situaría los anteriores sustantivos en puntos diferentes del eje imaginario que constituye el continuum de un extremo al otro de los registros representado en la siguiente figura:

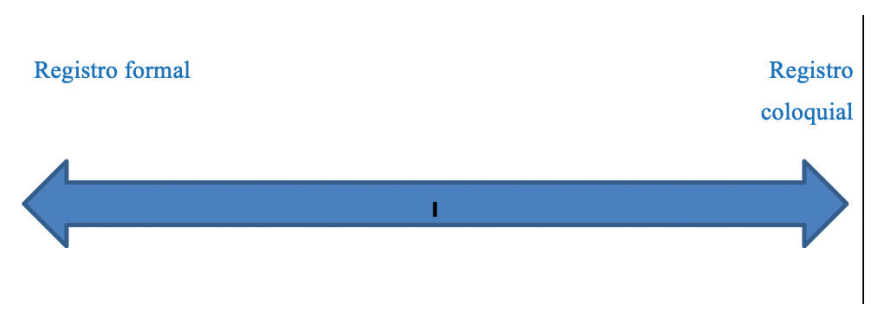

Figura 1. Eje de los registros

Estas herramientas para la descripción de los registros sirven para analizar datos referidos tanto a una L1 como una L2. En cuanto a la L2, presentan información de utilidad para la descripción de la interlengua y el análisis contrastivo. Cabe señalar que la utilidad de dicha descripción y de un análisis contrastivo profundo de los registros viene motivados por las diferencias que presentan el eje de los registros en diferentes lenguas.

En los siguientes apartados nos centraremos en el análisis de los pro-verbos mediantes dichas herramientas. Los pro-verbos se incluyen dentro de las palabras comodines, también llamadas pro-forma o palabras ómnibus, las cuales, debido a su significado difuso, sustituyen polisémicamente a amplios grupos de palabras. Gaviño Rodríguez (2008: 75) cita como ejemplo los pro-verbos hacer, tener, haber, poner, decir, echar o dar. Gómez Torrego (1995: 186-191) concluye que las pro-formas en general deben ser utilizadas exclusivamente en el registro coloquial. 


\section{El estudio}

En los siguientes apartados presentamos las características de la metodología y de los participantes del estudio.

\section{1.}

\section{Metodología del estudio}

Los sujetos participantes en el estudio recibieron una ficha como la presentada en la figura 2. En ella hay una serie de colocaciones propias del registro formal y pro-verbos, mostrados de manera aleatoria y formando pares ocultos. Así, por ejemplo, padecer una enfermedad es el equivalente formal de tener gripe o trabar amistad de hacer amigos.

El primer grupo de pares analizado corresponde al pro-verbo haber y sus equivalentes formales registrarse, percibirse y cometerse:

haber señales $\rightarrow$ percibirse indicios

haber un asesinato $\rightarrow$ cometerse un crimen

haber lluvias $\rightarrow$ registrarse precipitaciones

El segundo grupo de pares analizado correspondiente al pro-verbo tener y sus equivalentes formales padecer, acusar y albergar:

tener nervios $\rightarrow$ acusar estrés

tener expectativas $\rightarrow$ albergar esperanzas

tener gripe $\rightarrow$ padecer una enfermedad

El tercer conjunto analizado es el correspondiente al pro-verbo dar y sus equivalentes formales aducir, propinar y otorgar:

dar una paliza $\rightarrow$ propinar golpes

dar un premio $\rightarrow$ otorgar un diploma

dar razones $\rightarrow$ aducir motivos

El cuarto y último grupo de pares corresponde al pro-verbo poner y sus equivalentes formales sintonizar, prestar y montar: 


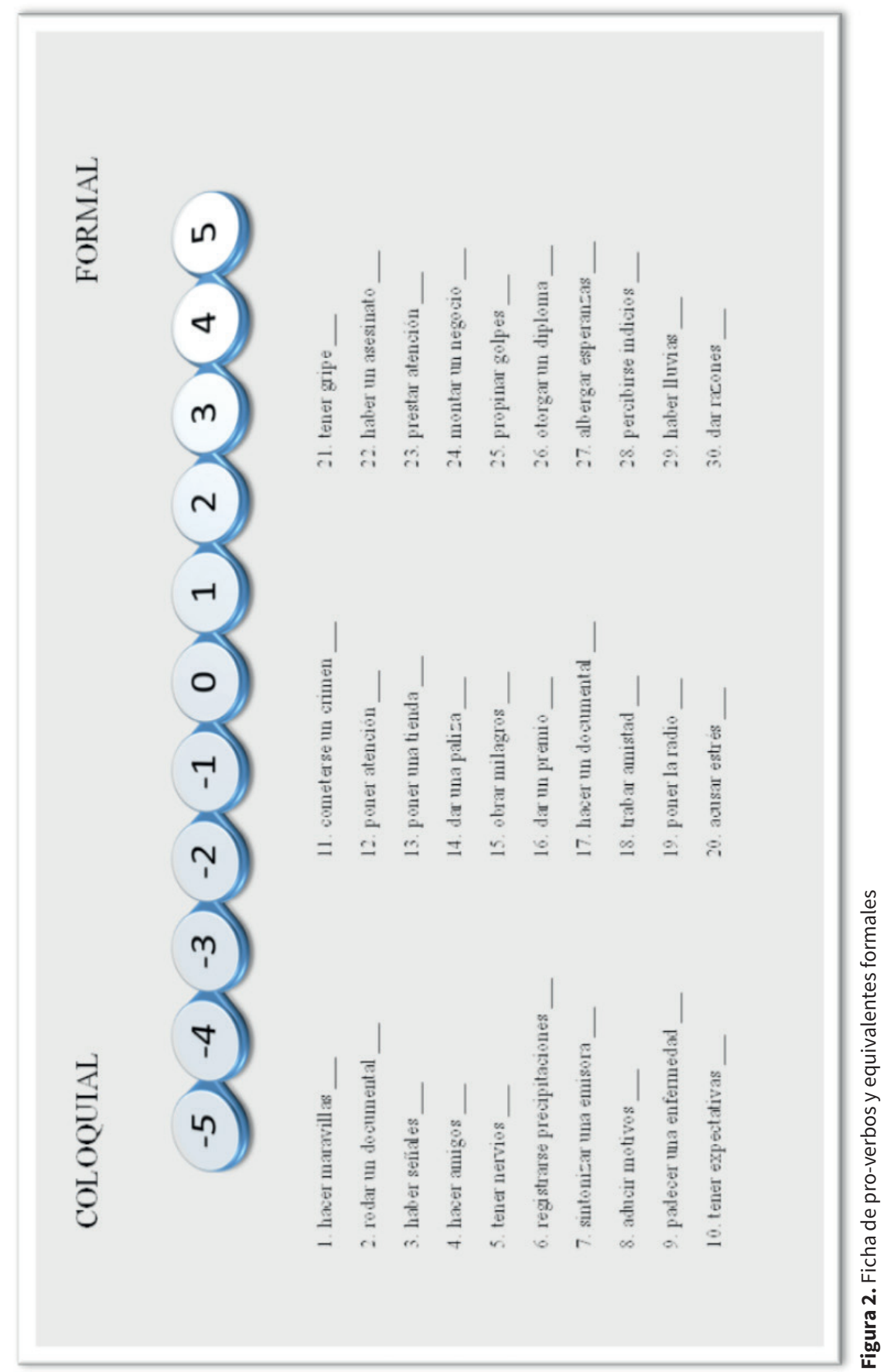

Reconocimiento y percepción del registro coloquial español... 
poner atención $\rightarrow$ prestar atención

poner una tienda $\rightarrow$ montar un negocio

poner la radio $\rightarrow$ sintonizar una emisora

Los sujetos debían puntuar cada colocación de 5 a -5, representando el 0 un valor neutro frente a 5, muy formal, y -5 , muy coloquial. En caso de que el aprendiz desconociera el significado de algún conjunto de palabras, debía dejar en blanco el casillero correspondiente. De esta manera se podía también evaluar el conocimiento del léxico en función de haber participado o no en el programa Erasmus.

\section{2.}

\section{Participantes}

El estudio ha contado con la participación de 50 sujetos, todos ellos estudiantes de Filología Hispánica de la Universidad Adam Mickiewicz de Poznan (Polonia), durante los cursos académicos 2015-2016 y 2016-2017, y cuyos resultados han sido divididos en tres grupos:

- El primero de ellos estaba formado por 21 estudiantes polacos (nivel C1, estudiantes del tercer curso de los Estudios de Grado), quienes han disfrutado de un semestre de estancia en una universidad española gracias al programa Erasmus y a los que denominaremos en adelante estudiantes $\mathrm{E}$.

- El segundo grupo estaba formado por 24 estudiantes polacos (nivel C1, estudiantes del tercer curso de los Estudios de Grado), quienes no han disfrutado de un semestre de estancia en una universidad española gracias al programa Erasmus y a los que denominaremos en adelante estudiantes NoE.

- El tercero de ellos, al cual debemos tratar como grupo de control, estaba formado por 5 nativos españoles, los cuales realizaban estancias de estudio en Polonia.

Cabe mencionar que la cifra total de 50 participantes puede considerarse como poco representativa, sobre todo en el caso del grupo de control. Aun así, nos gustaría remarcar que el número de participantes aumentará durante los próximos años hasta que 
cada uno de los tres grupos esté formado por 50 sujetos. No obstante, debido a la uniformidad de las respuestas, no consideramos que en el caso que nos atañe en este artículo las conclusiones varíen con un número mayor de sujetos de estudio.

\section{4.}

\section{Análisis de los datos}

En los siguientes apartados presentamos los resultados referidos a los pro-verbos analizados, los cuales, como ya hemos mencionado antes, son aquellos que por su significado difuso, sustituyen polisémicamente a amplios grupos de palabras.

\section{1.}

\section{El pro-verbo haber}

El primer grupo analizado es el correspondiente al pro-verbo $h a-$ ber y sus equivalentes formales registrarse, percibirse y cometerse.

\section{Haber}

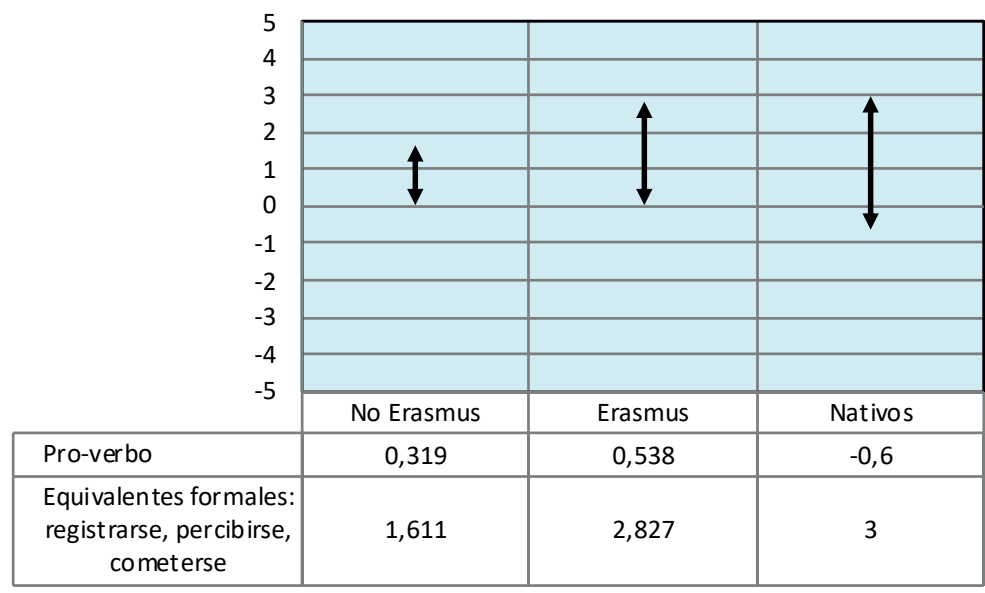

Figura 3. Reconocimiento y percepción del verbo haber y equivalentes formales 
Si observamos la figura 3, comprobaremos que la parte inferior de las flechas, correspondientes a los datos obtenidos de los pro-verbos, se mueven entorno a valores próximos al 0 . No obstante, los datos de los dos grupos de aprendices polacos tienen valores positivos, mientras que el grupo de control sitúa los pro-verbos dentro del ámbito de lo coloquial $(-0,6)$.

En cambio, si observamos la parte superior de las flechas, correspondientes a los datos obtenidos de los equivalentes formales, comprobaremos cómo los resultados de los estudiantes E tienen unos resultados similares a los de los nativos, mientras que los estudiantes NoE perciben los verbos formales como formas más neutras.

Es decir, para los estudiantes NoE la distancia entre el pro-verbo haber y sus equivalentes formales es mucho menor que la presentada por los estudiantes E y el grupo de control.

En cuanto al conocimiento léxico, cabe mencionar que el hecho de haber disfrutado de una estancia en un país hispanohablante no afecta sustancialmente y muestra niveles muy similares, tal y como se observa en la figura 4.

\section{Haber}

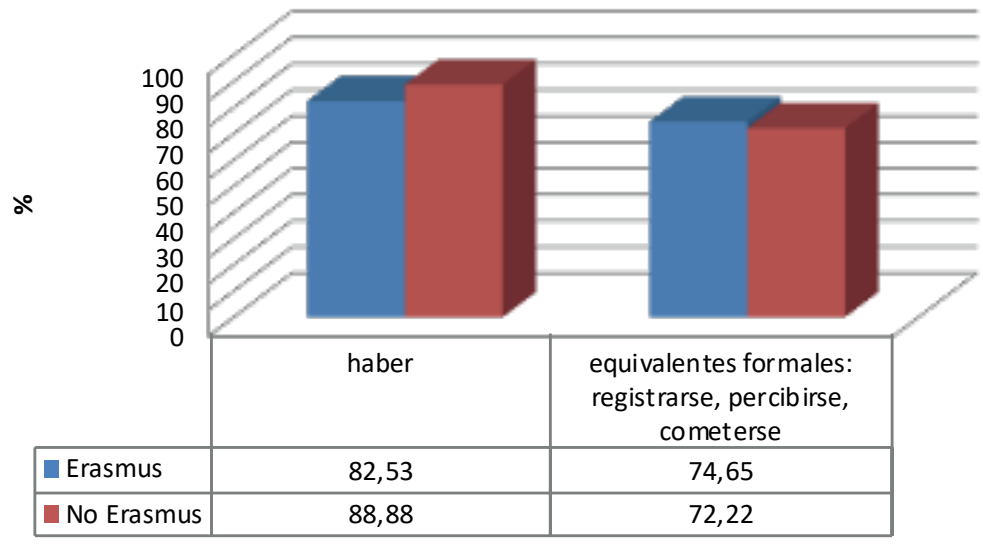

Figura 4. Conocimiento del verbo haber y equivalentes formales 


\section{2. \\ El pro-verbo tener}

El segundo grupo analizado es el correspondiente al pro-verbo tener y sus equivalentes formales padecer, acusar y albergar.

\section{Tener}

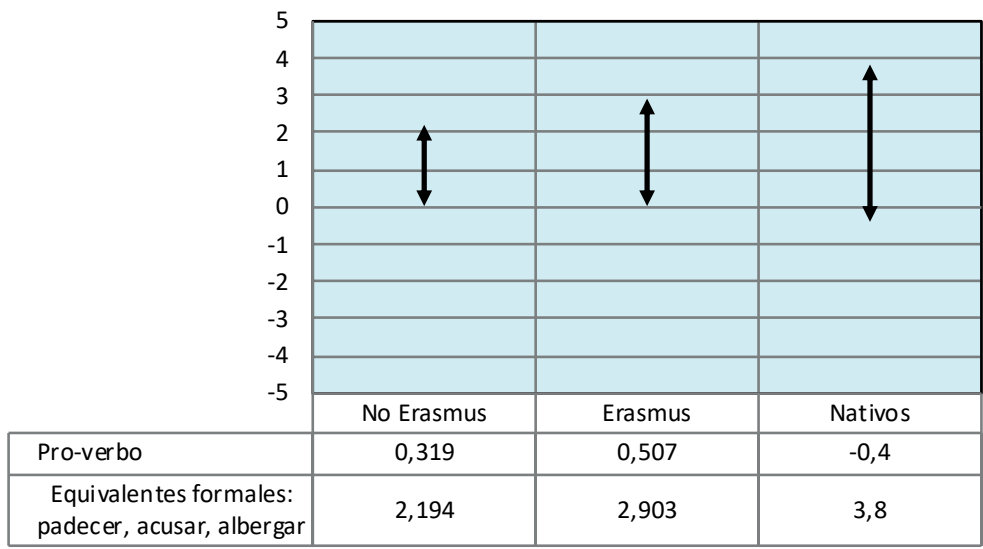

Figura 5. Reconocimiento y percepción del verbo tener y equivalentes formales

Si observamos la figura 5, comprobaremos que la parte inferior de las flechas, correspondientes a los datos obtenidos de los pro-verbos, se mueven entorno a valores próximos al 0 . No obstante, los datos de los dos grupos de aprendices polacos tienen valores positivos, mientras que el grupo de control sitúa los pro-verbos dentro del ámbito de lo coloquial $(-0,4)$.

En cambio, si observamos la parte superior de las flechas comprobaremos como los resultados de los nativos estudiantes muestran una clara identificación de los verbos padecer, acusar y albergar como formales. Sin embargo, nuevamente los NoE los perciben más próximos a valores neutros. Nuevamente, los estudiantes E tienden a separar ambos registros, aproximándose a los datos del grupo de control.

En cuanto al conocimiento léxico, como en el caso anterior, no hay sustanciales diferencias según se haya o no estado en un país hispanohablante, tal y como se observa en la figura 6 . 


\section{Tener}

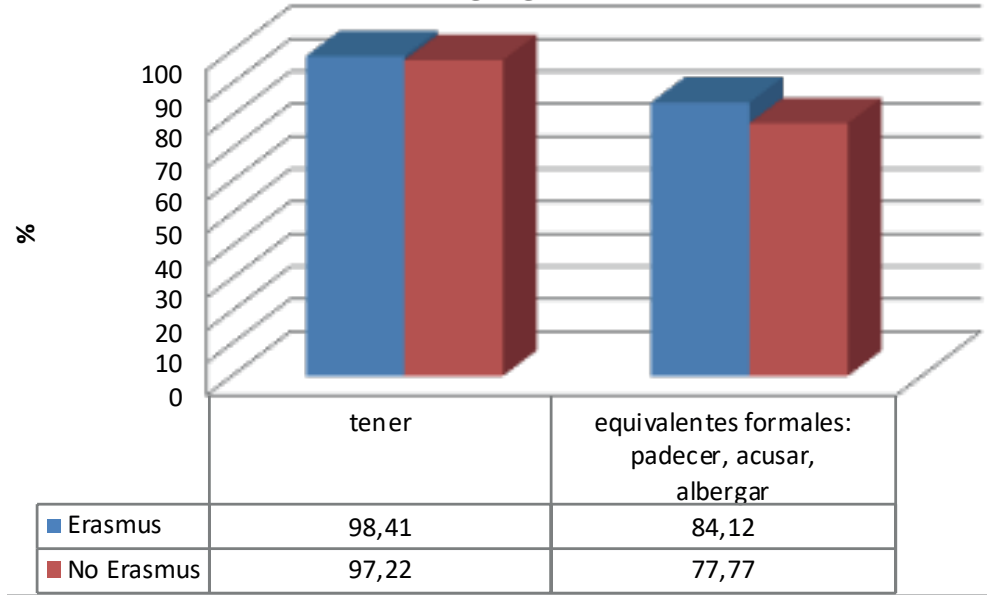

Figura 6. Conocimiento del verbo tener y equivalentes formales

\section{3.}

\section{El pro-verbo dar}

El tercer conjunto analizado es el correspondiente al pro-verbo dar y sus equivalentes formales aducir, propinar y otorgar.

\section{Dar}

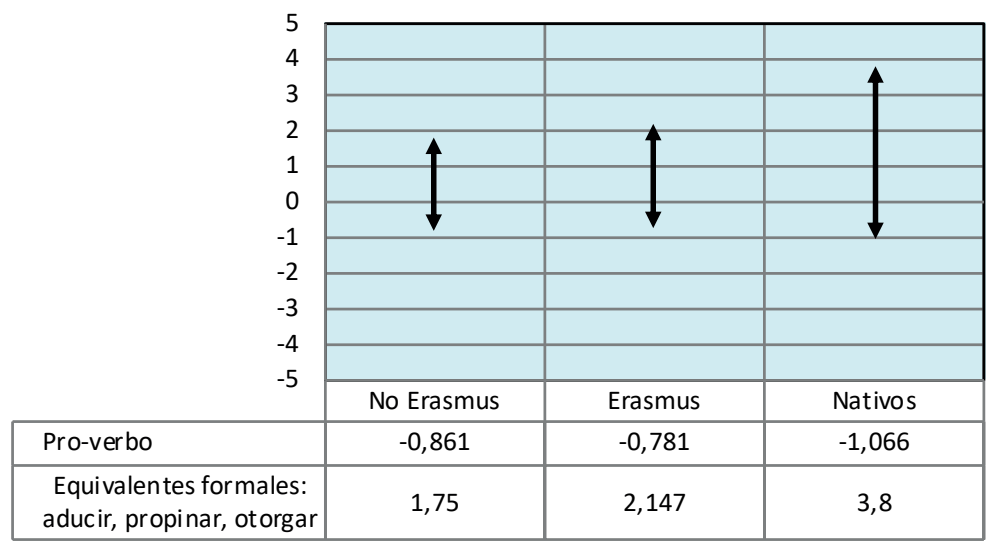

Figura 7. Reconocimiento y percepción del verbo dar y equivalentes formales 
En este caso los tres grupos de sujetos reconocen los pro-verbos como coloquiales. Sin embargo, el grupo de control los perciben con una coloquialidad mayor, así como nuevamente percibe con un mayor grado de formalidad los verbos aducir, propinar y otorgar. Es decir, la distancia entre registros está mucho más marcada para los nativos que para los aprendices polacos. Aun así, los estudiantes $\mathrm{E}$ muestran un ligero aumento en la distancia entre registros frente a los estudiantes NoE.

El conocimiento léxico, como muestra en la figura 8 y ya ocurría en los conjuntos arriba analizados, no muestra diferencias significativas en función de las estancias en un país hispanohablante.

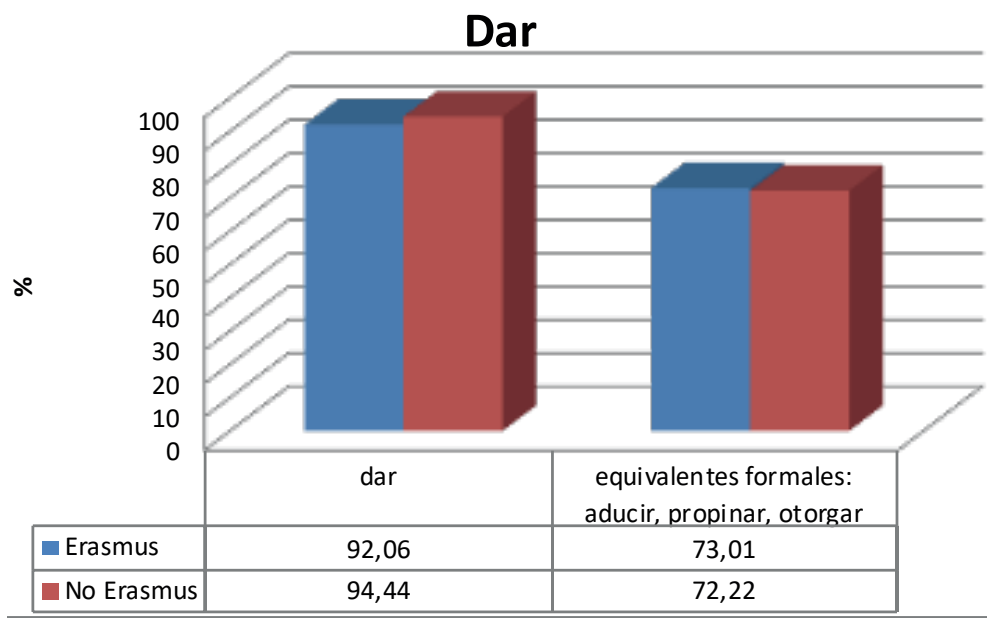

Figura 8. Conocimiento del verbo dar y equivalentes formales

\section{4. \\ El pro-verbo poner}

El cuarto y último grupo analizado es el correspondiente al pro-verbo poner y sus equivalentes formales sintonizar, prestar y montar.

La dinámica mostrada en este grupo es similar a los anteriores. En primer lugar, el grupo de control distingue claramente entre los dos registros. En cambio, los dos grupos de aprendices polacos sitúan el pro-verbo en valores neutros. Sin embargo, los equivalentes formales se reconocen como tales, e incluso el grupo 
E otorga un valor de formalidad superior al otorgado por el grupo de control.

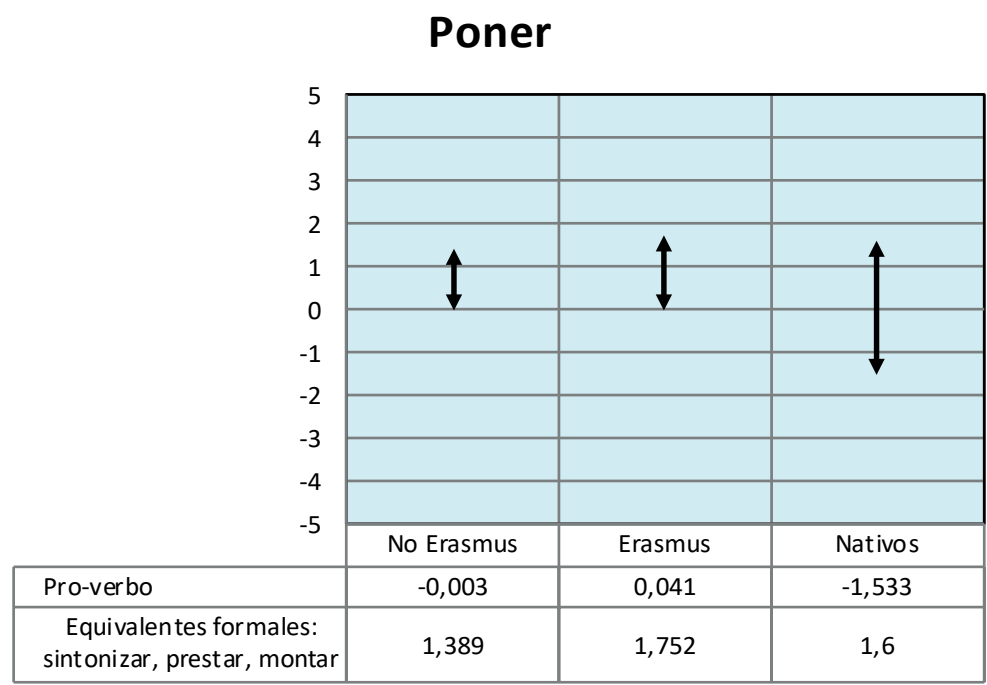

Figura 9. Reconocimiento y percepción del verbo poner y equivalentes formales

En cuanto al conocimiento léxico, tal y como muestra la figura 10, la diferencia no es sustancial entre aquellos sujetos que han realizado una estancia en un país hispanohablante y los que no la han realizado.

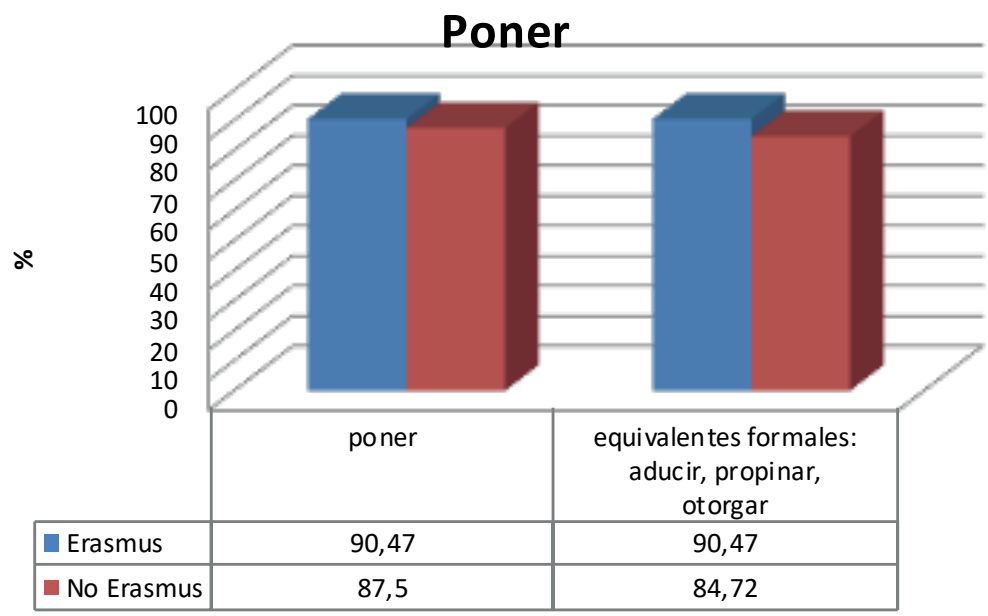

Figura 10. Conocimiento del verbo poner y equivalentes formales 


\section{5.}

\section{En conjunto}

En el presente apartado nos gustaría mostrar el resultado conjunto de la suma de los datos obtenidos de los cuatro grupos analizados.

Tal y como hemos observado, la dinámica presentada en todos los conjuntos es sumamente similar. Por una parte, el grupo de control distingue claramente entre la coloquialidad de los pro-verbos y la formalidad de las colocaciones. Sin embargo, los aprendices polacos no reconocen la coloquialidad de los pro-verbos y los consideran formas neutras, otorgándoles un valor casi 0 . Obsérvese la figura 11.

No obstante, y en referencia únicamente a los equivalentes formales, los sujetos E presentan unos valores que tienden a aproximarse a los del grupo de control. Mientras que los sujetos NoE los perciben en un grado de formalidad muy inferior al de dicho grupo.

En definitiva, mientras que el grupo de NoE presenta una distancia de 1,679 puntos entre ambos registros, en el grupo E es de 2,331, todavía lejos de los 3,949 puntos del grupo de control, pero con una clara tendencia aproximativa.

\section{Totales}

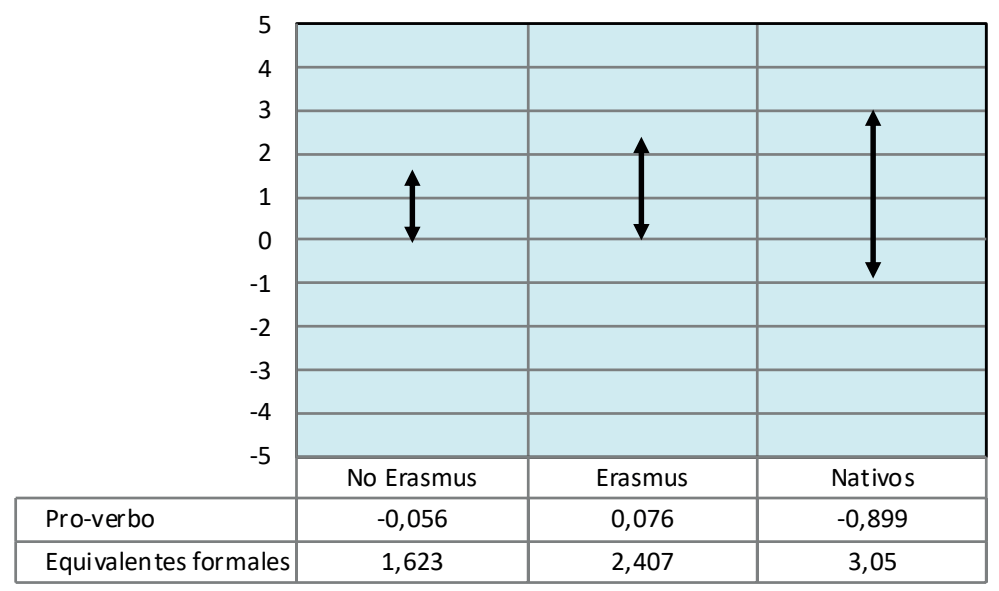

Figura 11. Reconocimiento y percepción de los pro-verbos y equivalentes formales 
En cambio, si bien una estancia prolongada en un país de habla española puede influir en la percepción de los verbos, no se puede afirmar lo mismo respecto al conocimiento del léxico, puesto que este no varía sustancialmente.

\section{Totales}

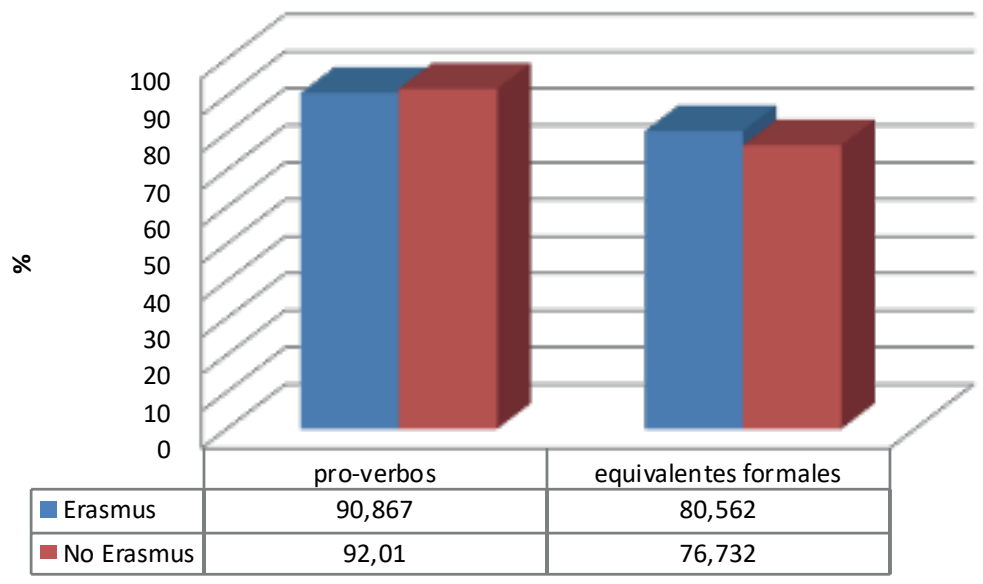

Figura 12. Conocimiento de los pro-verbos y equivalentes formales

\section{4.}

\section{Conclusiones}

Después de analizar los diferentes conjuntos de pro-verbos y sus equivalentes formales las conclusiones a las que llegamos son las siguientes:

En primer lugar, los nativos distinguen claramente entre pro-verbos, pertenecientes al registro coloquial, y los verbos equivalentes del registro formal. En cambio, tanto los estudiantes E como los NoE perciben los pro-verbos como formas neutras. Probablemente se deba a que son formas que no asocian con registros sino con las primeras etapas de aprendizaje del español, en la que los pro-verbos se usan por su mayor recursividad y se aprenden en contexto académico, no vinculado por tanto con el registro coloquial. 
En segundo lugar, la percepción de la distancia entre registros es mayor en los estudiantes E que en los NoE, aproximándose a la de los nativos, aunque sin alcanzar los mismos resultados, puesto que, como mencionábamos arriba, entienden los pro-verbos como neutros. Aun así, cabe hacer mención que el programa Erasmus ayuda a percibir los registros de una manera más cercana a la de los nativos.

En último lugar, el programa Erasmus no provoca un aumento significativo del conocimiento del léxico analizado. Por lo tanto, el conocimiento del léxico de los pro-verbos y sus equivalentes formales alcanzado en el ámbito académico en un país no hispanohablante es equiparable al alcanzado en el ámbito académico del país cuya lengua se estudia.

\section{Referencias bibliográficas}

Anusiewicz, J. (1992), "Potoczność jako sposob doświadczania świata i jako postawa wobec świata”, en J. Anusiewicz, F. Nieckula (eds.), Język a kultura. T. 5: Potoczność w języku i w kulturze, Wrocław: Wydawnictwo Uniwersytetu Wrocławskiego, 9-20.

Bartmiński, J. (1991), "Styl potoczny jako centrum systemu stylowego języka”, Poradnik jezzkowy”, 1-2, 10-23.

Bartmiński, J. (1992), “Styl potoczny”, en J. Anusiewicz, F. Nieckula (eds.), Język a kultura. T. 5: Potoczność w języku i w kulturze, Wrocław: Wydawnictwo Uniwersytetu Wrocławskiego, 37-54.

Bartmiński, J. (1993), “Styl potoczny”, en J. Bartmiński (ed.), Wspołczesny język polski, Wrocław: Wydawnictwo Wiedza o Kulturze, 115-134.

Briz Gómez, A. (coord.) (1995), La conversación coloquial. Materiales para su estudio, Valencia: Universitat de València.

Briz Gómez, A. (1996), El español coloquial. Situación y uso, Madrid: Arco Libros.

Briz Gómez, A., Grupo Val.Es.Co. (2002), Corpus de conversaciones coloquiales, Madrid: Arco Libros.

Gaviño Rodríguez, V. (2008), Español coloquial. Pragmática de lo cotidiano, Cádiz: Servicio de publicaciones de la Universidad de Cádiz. 
Gómez Torrego, L. (1995), El léxico en el español actual: uso y norma, Madrid: Arco Libros.

Hołowka, T. (1986), Myślenie potoczne. Heterogeniczność zdrowego rozsądku, Varsovia: Państwowy Instytut Wydawniczy.

Real Academia Española (s.f.), Diccionario de la lengua española, [en línea] <http://www.rae.es>, [fecha de consulta: 10.02.2018].

Zdunkiewicz-Jedynak, D. (2008), Wykłady ze stylistyki, Varsovia: Wydawnictwo Naukowe PWN. 